\title{
Case report of tubercular spondylodiscitis with paraplegia managed by posterior transpedicular decompression and pedicle screw fixation ${ }^{*}$
}

\author{
Paragjyoti Gogoi $^{1 \#}$, Anshuman Dutta $^{1}$, Vikash Agarwala $^{1}$, Prasanta Sonowal $^{2}$ \\ ${ }^{1}$ Department of Orthopaedics \& Trauma, Silchar Medical College, Silchar, India; ${ }^{\#}$ Corresponding Author: pggogoiparag@gmail.com \\ ${ }^{2}$ Department of Anaesthesiology, Silchar Medical College, Silchar, India
}

Received 20 May 2013; revised 21 June 2013; accepted 25 July 2013

Copyright (C) 2013 Paragjyoti Gogoi et al. This is an open access article distributed under the Creative Commons Attribution License, which permits unrestricted use, distribution, and reproduction in any medium, provided the original work is properly cited.

\begin{abstract}
Pott's paraplegia is still prevalent in this part of the world. Early onset paraplegia can be improved by timely surgical intervention under ATT cover. The disease mostly affects the thoraco-lumbar spine. Classically, the diseased area is addressed by anterior thoracic or thoracolumbar approach and after curettage of the diseased and necrotic material the anterior column is reconstructed by rib or fibular strut graft or metallic cage and supplemented by posterior instrumentation and fusion. Laminectomy, as a method of decompression, was greatly discouraged in spinal tuberculosis with compressive myelopathy except in posterior element involvement. We present a case of a 35 years old lady with Pott's paraplegia treated by hemilaminectomy and transpedicular limited anterior decompression of the cord and pedicle screw fixation with fusion who improved vastly in terms of motor power.
\end{abstract}

Keywords: Spinal TB; Pott's Paraplegia; Adult; Hemi-Laminectomy; Pedicle Screw; Fusion

\section{INTRODUCTION}

Spinal tuberculosis is still a cause of major morbidity. Millions of people are still affected by this ailment. Because of the improved chemotherapy the mortality is now reduced to a great extent. Many cases got improvement by chemotherapy alone. Only some selected cases require surgical treatment. Spinal tuberculosis is notorious for producing spinal deformities and neurological

${ }^{*}$ Consent: Informed consent obtained from the patient regarding presentation and publication of this case. involvement like paraplegia. In such cases immediate surgical intervention becomes necessary to regain normal or useful motor function [1].

Pott's paraplegia can occur due to compression of the spinal cord by soft material like tubercular abscess, caseous mass or granulation tissue or by hard material like internal gibbus, bony sequestrum or a sequestrated disc.

We report a case of Spinal tuberculosis at D12 and L1 level presenting with paraplegia with bowel and bladder involvement who regained completely normal motor function and bowel and bladder control after posterior decompression and stabilization with pedicle screw rod system.

\section{THE CASE REPORT}

A lady of 35 years old presented to us with complete loss of all motor function of her both lower limbs with retention of urine and pain over the lower dorsal spine. She had a history of back pain for one month associated with fever off and on. She did not sustain any trauma over the area nor was there any history of cough for prolonged duration or significant weight loss.

On examination both the lower limbs were flaccid with grade 0 motor power according to MRC grading. She did not feel the sensation of bladder fullness. Her sensation was diminished from L1 dermatome.

The spine examination revealed a kyphus deformity at D12 and L1 level with mild tenderness. Wasting of paraspinal muscles were also noted. Plain X-ray showed obliteration of D12, L1 disc space along with destruction of inferior part of body of D12 and superior part of body of L1 as well as wedging at that level. No obvious paraspinal soft tissue shadow was noted.

MRI scan of the Dorso-lumbar Spine confirmed the $\mathrm{X}$-ray findings (Figures 1 and 2). There was destruction of the vertebrae with compression of the spinal cord at 
D12 and L1 level. Mild abscess noted in and around the destructed vertebrae. Pedicles were normal at all levels.

Blood examination showed increase ESR level and lymphocytosis. Her liver and renal function tests were within normal limits.

From the clinical history, physical examination and relevant investigation findings the diagnosis of Pott's paraplegia was made and four drugs combination chemotherapy started. Early surgical intervention was planned to decompress the cord and stabilize the spine.

The spine was exposed from the posterior aspect. It was then stabilized with four pedicle screws, two at D11 level and two at L2 level. Hemilaminectomy done at D12 vertebra on right side and the right pedicle was gradually removed with rongeours and nibblers. The cord was decompressed anteriorly by an angulated rongeour removing the diseased material partly from the D12 body. Collectively the cord was decompressed anteriorly, laterally and partly posteriorly. Posterolateral fusion with bone graft was undertaken and the surgical wound was closed in layers.

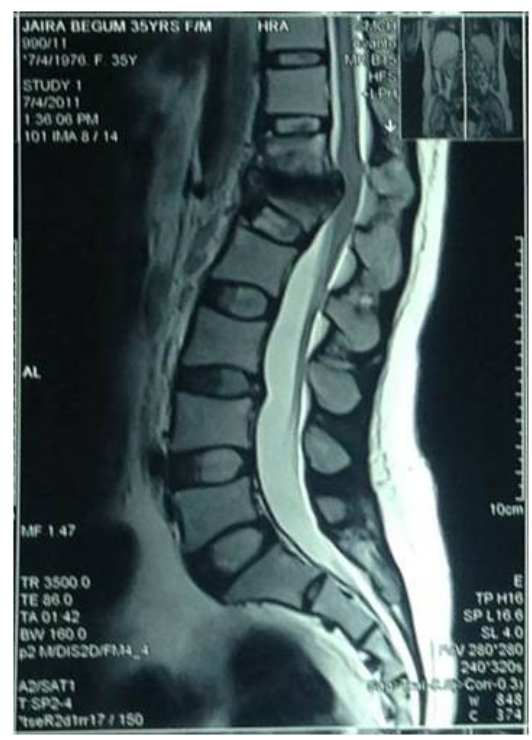

Figure 1. MRI scan of the affected spine.

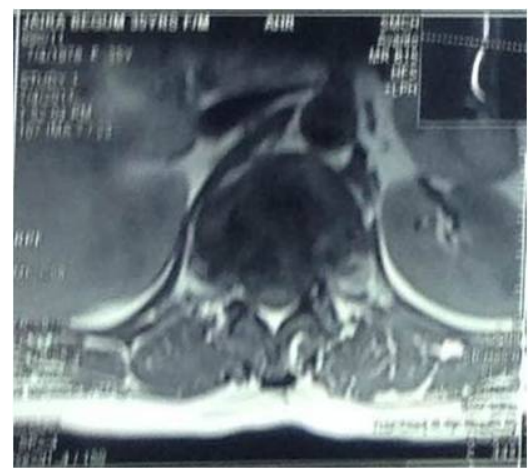

Figure 2. MRI scan of the affected spine.
Post-operatively the patient was continued with chemotherapy along with assisted physiotherapy. Her motor power gradually improved after the surgery and she regained motor power of grade 3 after four weeks and grade 4 after eight weeks. Her bladder control came back after four weeks and she started walking with walker from eight weeks with a Taylor brace. Her back pain resolved and no episode of fever was noted after the operation (Figure 3).

After about eight months following the surgery her pedicle screws started to back out with pseudoarthosis at the fused level (Figure 4). However there was no deterioration in her neurological status. The pedicle screws were revised incorporating one more level below (Figure 5). Six months down the line, the patient is doing well. There is no pain at the back; no sign of screw back out. Fusion is still doubtful, but the anterior column structures healed completely and there is no progression of the kyphotic angle (Figure 6).

\section{DISCUSSION}

Surgical treatment for spinal tuberculosis with paraplegia is constantly evolving. Neurological involvement warrant early surgical intervention. In earlier days the spinal canal was decompressed by simple laminectomy

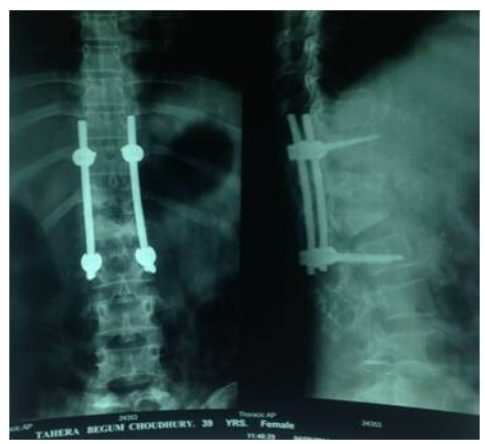

Figure 3. Post-operative X-ray after the first surgery.

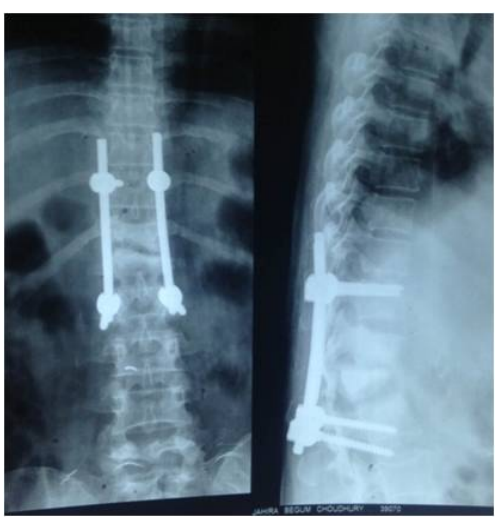

Figure 4. Development of pseudo-arthrosis and Screw backout at 8 months. 


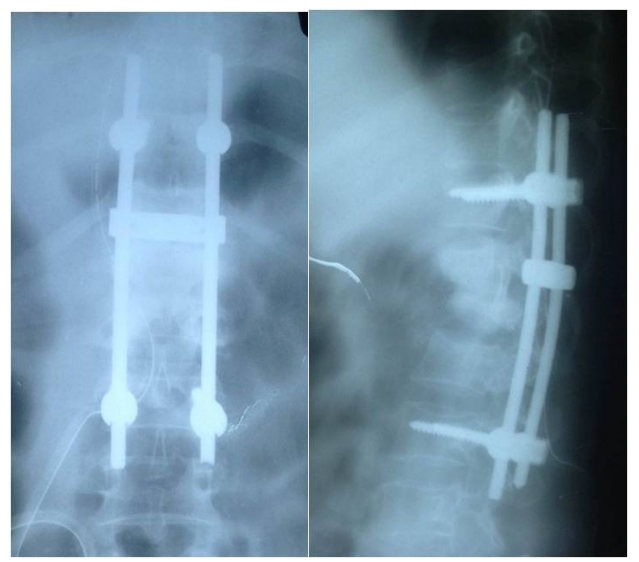

Figure 5. After revision surgery.

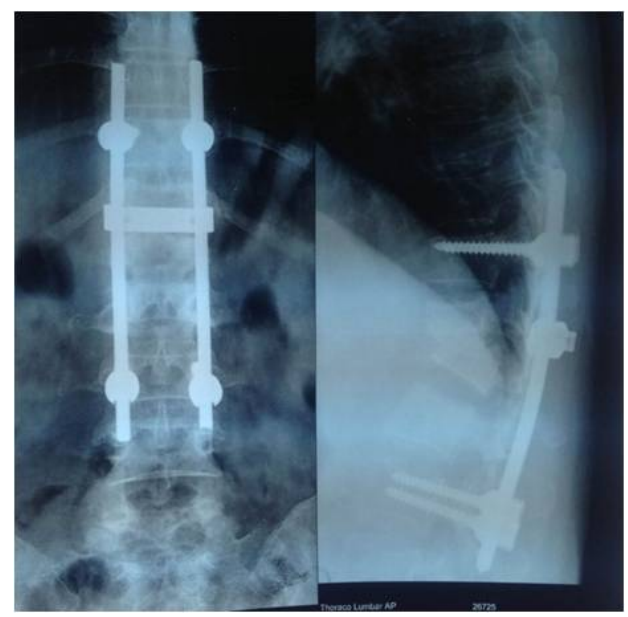

Figure 6. Six months follow up X-ray after the revision surgery.

via posterior approach. Patients used to recover from the neural compression but the spine became unstable. As majority of spinal tuberculosis involve and destruct the anterior column, the only stabilizing structure preventing the kyphosis remained the posterior elements. So, laminectomy further destabilizes the spinal column. For this reason and lack of proper instrumentation from the posterior aspect laminectomy was disfavored later on. Seddon even condemned laminectomy as a surgical procedure for spinal tuberculosis [2].

Hemilaminectomy or removal of half of the lamina to decompress the spinal canal was used for easy posterior approach as well as lesser destabilizing effect on the spinal column.

Costo-transversectomy and antero-lateral decompression were two procedures slowly gaining popularity [3]. Costo-transversectomy is a simple procedure for drainage of the abscess in thoracic region. In the antero-lateral decompression procedure the spinal canal is decompressed by sacrificing the pedicle as well as the anterior diseased material. They do not address the radical re- moval of diseased vertebrae or reconstruction of anterior column; hence nothing to do with spinal stability or progression of the deformity.

Hodgeson et al. during their Hongkong experience devised the radical excision of the diseased area by anterior thoracic or thoraco-lumbar approach and reconstructing the anterior column by rib graft. They noted significant improvement in terms of mortality, spinal stability and cure from the infection [4].

Rajasekaran and Soundarapandian noted failure of rib graft and progression of kyphosis following the Hongkong procedure [5]. They reported fibular graft as better alternative. In another study they proposed a formula for expected progression of the kyphotic angle from the initial radiographic measurement of destruction [6].

Anterior column is reconstructed by rib grafts, fibular strut graft, metallic cage along with anterior instrumentation like locking plates. They can be supplemented by posterior instrumentation and fusion.

Posterior stabilization was earlier done by closed rectangles with sublaminar wires; rod and laminar hook constructs like Herrington's, Luque rod system [7]. Presently these are mostly replaced by pedicle screw-rod system. All of them act as a tension band in stabilizing the spine; so the anterior column continuity is a prerequisite for them.

Anterior column reconstruction from the posterior transpedicular approach has slowly gained popularity. The diseased material is curetted from the posterior aspect, the cord got decompressed from both anterior and lateral aspect and expandable cage fills the anterior column defect. Posterior instrumentation then stabilizes the spine. This procedure obviates the morbidity associated with anterior thoracotomy approach. This approach is utilized in vertebral osteomyelitis [8], osteoporotic compression fractures [9], metastatic spinal tumours [10].

Mehta and Bhojraj proposed a MRI dependent classification system of spinal tuberculosis where they categorized them into four groups. They advocated posterior transpedicular decompression in the group who cannot tolerate the thoracotomy [11]. Guven et al. also showed good result in their cases treated by this approach [12].

Posterior instrumentation was chosen in our patient as they are familiar to us as a routine procedure in traumatic spine stabilizations. Pedicle screws offer sufficient stabilizing power and hemilaminectomy along with transpedicular limited anterior decompression ensures spinal cord relief.

Good chemotherapy helps in rapid healing of the anterior column and gives anterior support. Kyphosis progression usually stops after healing of the anterior column and posterior fusion. In our case kyphosis progressed and fusion failed resulting in pseudoarthrosis as there was no adequate anterior support during the healing 
period. Revised posterior instrumentation along with fusion is doing well because of anterior support provided by the healed anterior structures which were clinically confirmed during revision surgery.

\section{CONCLUSION}

Posterior transpedicular limited anterior decompression is a viable option in Pott's paraplegia. However, anterior column reconstruction is important in preventing kyphosis progression. Pseudoarthrosis may be present in infective spinal conditions also.

\section{REFERENCES}

[1] Tuli, S.M. (1997) Tuberculosis of the skeletal system: Bones, joints, spine and bursal sheaths. 2nd Edition, Jaypee Brothers, Bangalore.

[2] Seddon, H.J. (1935) Pott's paraplegia: Prognosis and treatment. British Journal of Surgery, 22, 769-799. doi:10.1002/bjs.1800228813

[3] Capener, N. (1954) The evolution of lateral rhachiotomy. Journal of Bone \& Joint Surgery, 36B, 173-179.

[4] Hodgson, A.R. and Stock, F.E. (1960) Anterior spine fusion for the treatment of tuberculosis of the spine. Journal of Bone \& Joint Surgery, 42A, 295-310.

[5] Rajasekaran, S. and Soundarapandian, S. (1989) Progression of kyphosis in tuberculosis of the spine treated by anterior arthrodesis. Journal of Bone \& Joint Surgery, 71A, 1314-1323.

[6] Rajasekaran, S. (1987) Prediction of the angle of gibbus deformity in tuberculosis of the spine. Journal of Bone \& Joint Surgery, 69A, 503-509.

[7] Dove, J. (1987) Luque segmental spinal instrumentation: The use of the Hartshill rectangle. Orthopedics, 10, 955961.

[8] Lu, D.C., Wang, V. and Chou, D. (2009) The use of allograft or autograft and expandable titanium cages for the treatment of vertebral osteomyelitis. Neurosurgery, 64, 122-129. doi:10.1227/01.NEU.0000336332.11957.0B

[9] Mochida, J., Toh, E., Chiba, M. and Nishimura, K. (2001) Treatment of osteoporotic late collapse of a vertebral body of thoracic and lumbar spine. Journal of Spinal Disorders, 14, 393-398. doi:10.1097/00002517-200110000-00004

[10] Bilsky, M.H., Lis, E. and Raizer, J. (1999) The diagnosis and treatment of metaststic spinal tumour. Oncologist, 4, 459-469.

[11] Mehta, J.S. and Bhojraj, S.Y. (2001) Tuberculosis of the thoracic spine. A classification based on the surgical strategies. Journal of Bone \& Joint Surgery, 83, 859-863. doi:10.1302/0301-620X.83B6.11142

[12] Guven, O., Kumano, K. and Yalcin, S. (1994) A single stage posterior approach and rigid fixation for preventing kyphosis in the treatment of spinal tuberculosis. Spine, 19, 1039-1043. doi:10.1097/00007632-199405000-00007 